\section{Military Technical College Kobry El-Kobbah, Cairo, Egypt}

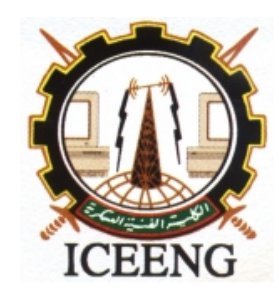

\section{$7^{\text {th }}$ International Conference on Electrical Engineering \\ ICEENG 2010}

\title{
On-line Control Technique for Missile Autopilot
}

\author{
By \\ Magdy Abo-Elela* \\ Ayman El-Shabrawy** \\ Ibrahim Hassan ***
}

\section{Abstract:}

In this paper a new learning strategy is used for on-line tuning the control system of the ballistic missile. The gradient descent method technique is utilized to derive the on-line tuning laws of the robust controller, so that the robust performance of the system can be yielded. As the result of using this method, the old technique "gain schedule" that normally used in ballistic missile is replaced. The simulation result shows a good settling time, less or no overshoot and higher robustness were achieved. Moreover, the new tuning process is successful in the presence of high noise.

\section{Keywords:}

PID tuning, Missile control system and Gradient descent

* College of Engineering, Cairo University, Cairo, Egypt

** Egyptian Armed Forces

*** Egyptian Armed Forces 


\section{Introduction:}

Due to its simplicity, reliability, and the clear relationship between its parameters and the system response specifications, the conventional PID control is still the most popular design approach used in the field of real time control even with the presence of the modern good sophisticated control scheme.

It is well known that a well-tuned PID controller is able to achieve an excellent performance. However, it suffers a main disadvantage of getting a poor performance whenever the plants are subjected to some kind of disturbance or the plants have a high order non-linear structure. Ballistic missiles undergo a dynamic change along the trajectory due to huge amount of the fuel flow rate. This condition and the presence of gusts result in an angle of attack not being zero at all time, however, the angle of attack must be kept small to avoid excessive loading of structure.

Although the PID controller have many advantage when it is used to control the ballistic missile, the tuning of a conventional PID controller has been becoming a challenging problem due to highly coupled non-linearity and parameters variations in the missile [6]. In order to deal with the problem, self-tuning PID control for ballistic missile is introduced.

In this paper, we propose a new self-tuning PID control for ballistic missile. Using the method of gradient descent optimization to the parameter space, adaptive algorithms for the gains of PID control are developed. Therefore, the controller parameters are adjusted automatically online. This makes the controller robust to the changes of missile parameters. Furthermore, this control approach brings the missile to a desired reference trajectory. In addition, since the only needed information is the output signal of the controlled plant, the proposed method is easy to implement. Theoretical analysis and extensive simulation results will be illustrated in following sections.

\section{Dynamic Model for a Ballistic-Type Missile}

The trajectory of ballistic-type missiles is planned to maintain the missile at a zero angle of attack [6]. This is normally attempted by programming the pitch attitude or pitch rate to yield a zero-g trajectory.

The $X$ axis of the missile is along the longitudinal axis of the missile, this body axis system becomes a stability axis system. By placing the $\mathrm{Z}$ axis in the trajectory plane, all pitch motion is about the $\mathrm{Y}$ axis (see Figure-1)

The equations describe the missile can be given as [6], 


$$
\begin{aligned}
& \left(\frac{m U}{S q} s-C_{z_{\alpha}}\right) \alpha(s)+\left(-\frac{m U}{S q} s-C_{w} \sin \Theta\right) \theta(s)=C_{z_{\delta}} \delta \\
& \left(-\frac{d}{2 U} C_{m_{\alpha \alpha}} s-C_{m_{\alpha}}\right) \alpha(s)+\left(\frac{I_{y}}{S q d} s^{2}-\frac{d}{2 U} C_{m_{q}} s\right) \theta(s)=C_{m_{\delta}} \delta
\end{aligned}
$$



Figure (1): Ballistic missile axis system

Where $m$ is missile mass, $d$ is missile diameter, $S$ is missile cross-sectional area, $\alpha$ is angle of attack, $\delta$ is thrust angle, $\theta$ is pitch angle, $U$ is linear velocity in OX axis, $u$ is change in linear velocity in $\mathrm{OX}$ axis, $q$ is dynamic pressure, $C_{z_{\alpha}}$ is variation of $\mathrm{Z}$ force with angle of attack, $C_{w}$ is gravity, $\Theta$ is angle between horizontal and OX axis measured in vertical plane, $C_{z_{\delta}}$ is change pitching moment due to change in thrust angle, $C_{m_{\delta}}$ is change in force in $\mathrm{Z}$ direction due to change in thrust angle, $C_{m_{\mathrm{d}}}$ is downwash lag on moment created by wings (for this missile equal zero), $C_{m_{\alpha}}$ is change in pitching moment due to a change in angle of attack, $I_{y}$ is moment of inertia in OT axis and $C_{m_{q}}$ is effect on pitching moment due to a pitch rate (damping in pitch).

The actuating signal $\delta$ is the deflection of the thrust chamber. The component of thrust normal to the $X$ axis is proportional to $\sin \delta$; however, if $\delta$ is small, $\sin \delta$ can be replaced by $\delta$ in radians. After $75 \mathrm{sec}$ equation (1) can be written as, 


$$
\begin{aligned}
& (88.5 s+3.13) \alpha(s)+(-88.5 s+2.06) \theta(s)=-4.63 \delta(s) \\
& -11.27 \alpha(s)+\left(4.75 s^{2}+0.321 s\right) \theta(s)=-34.25 \delta(s)
\end{aligned}
$$

Then the transfer function for $\delta$ input to $\theta$ output is

$$
\frac{\theta(s)}{\delta(s)}=\frac{-7.21(s+0.0526)}{(s+1.6)(s-1.48)(s-0.023)}
$$

This transfer function is terrifically varied with time, for example after $139 \mathrm{sec}$ the transfer function become

$$
\frac{\theta(s)}{\delta(s)}=\frac{-255.678 s}{s^{2}+0.884 s-0.9884}
$$

This shows the need for our proposed self tuning PID controller.

\section{Self-tuning PID Control Design}

The most popular controller used in missile is PID controller because the PID controller is simple and reliable to implement. However, because of both variation and high uncertainty of missile dynamics, tuning of PID control gains becomes a challenging problem. Normally the trajectory is divided into segments and each segment has different gains for the PID controller, this method is called "gain schedule". In this study, we use the concept of gradient descent optimization to develop a self-tuning PID controller, which is only dependent on the output (pitch angle).

First, we partition the whole system into four parts as shown in Figure 2, which is similar to the one given in [3]. Part 1 is the proportional part with the exception of proportional gain $K_{P}$. Part 2 is the integral part with the exception of integral gain $K_{I}$. Part 3 is the derivative part with derivative gain $K_{D}$ and part 4 is the controlled plant.

Then we consider $K_{P}, K_{I}$ and $K_{D}$ as independent variables. Based on the principle of gradient descent optimization, we define an objective function as

$$
E=\frac{1}{2} \varepsilon^{2}=\frac{1}{2}\left(\theta_{i}-\theta_{0}\right)
$$

By using the algorithm of gradient descent

$K^{\&}=-\lambda \frac{d E}{d K}$, where $K=\left\{K_{P}, K_{I}, K_{D}\right\}$ 


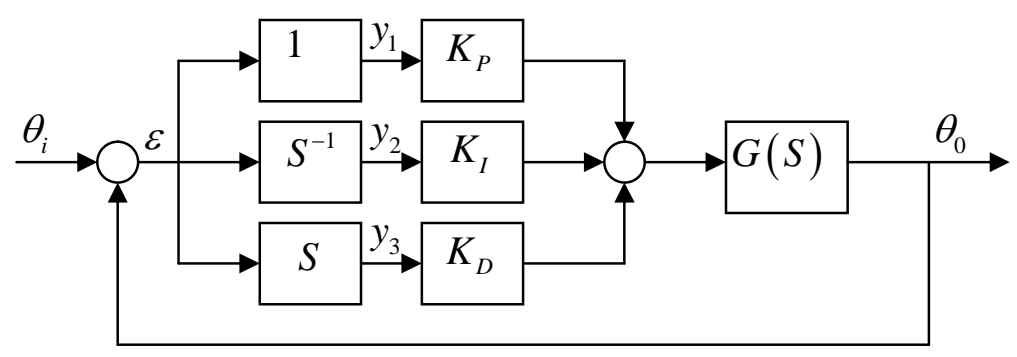

Figure (2): Design of self tuning PID controller for ballistic missile

By using the linearization of input-output algorithm in [7] and the assumption of Frechet derivative in [3], We then have

$$
I_{P}^{\&}=\eta_{1} \varepsilon y_{1}
$$

In which $\eta_{1}$ is a constant. Similarly we can obtain

$$
\begin{aligned}
& K_{I}^{\&}=\eta_{2} \varepsilon y_{2} \\
& K_{D}^{\&}=\eta_{3} \varepsilon y_{3}
\end{aligned}
$$

where $\eta_{2}$ and $\eta_{3}$ are constant.

Let $\eta_{1}=\eta_{2}=\eta_{3}=\eta$ in which $\eta$ is an adaptive coefficient. The control objective is to bring the output pitch angle $\theta_{0}$ to the desired pitch angle $\theta_{i}$ generated from guidance law using the tuning algorithm given by equation (6) and (7).

\section{Simulatiom Result:}

In order to evaluate and validate the effectiveness of our proposed control design presented in the previous sections, a simulation program has been developed by using the Matlab/ Simulink software. Figure 3 shows the block diagram of a ballistic missile system. The program is based on the structure shown in Figure 3. The figures show the different phases in trajectory. In each phase the PID is modified to follow the dynamic change of the missile, while the purposed controller is changing its gain automatically in each phase. 


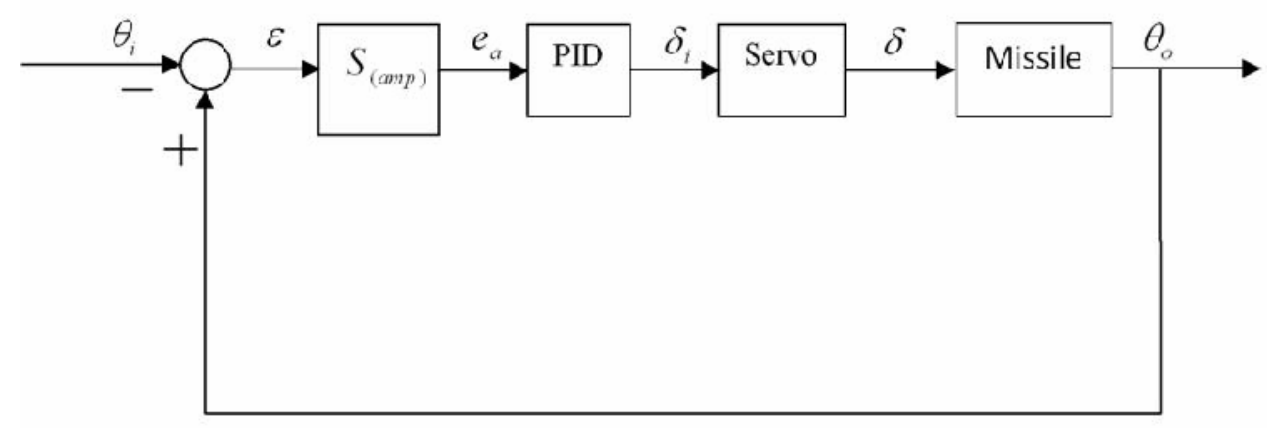

Figure (3): Missile pitch orientation control system

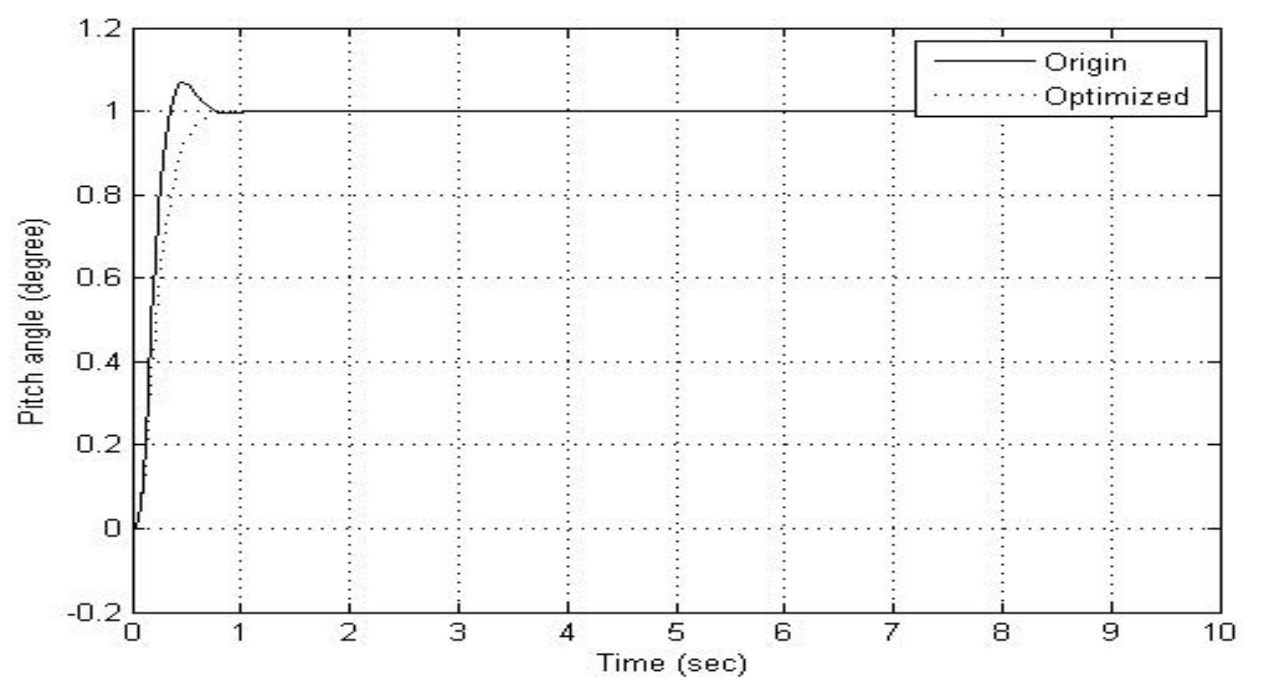

Figure (4): Missile response at $45 \mathrm{Sec}$

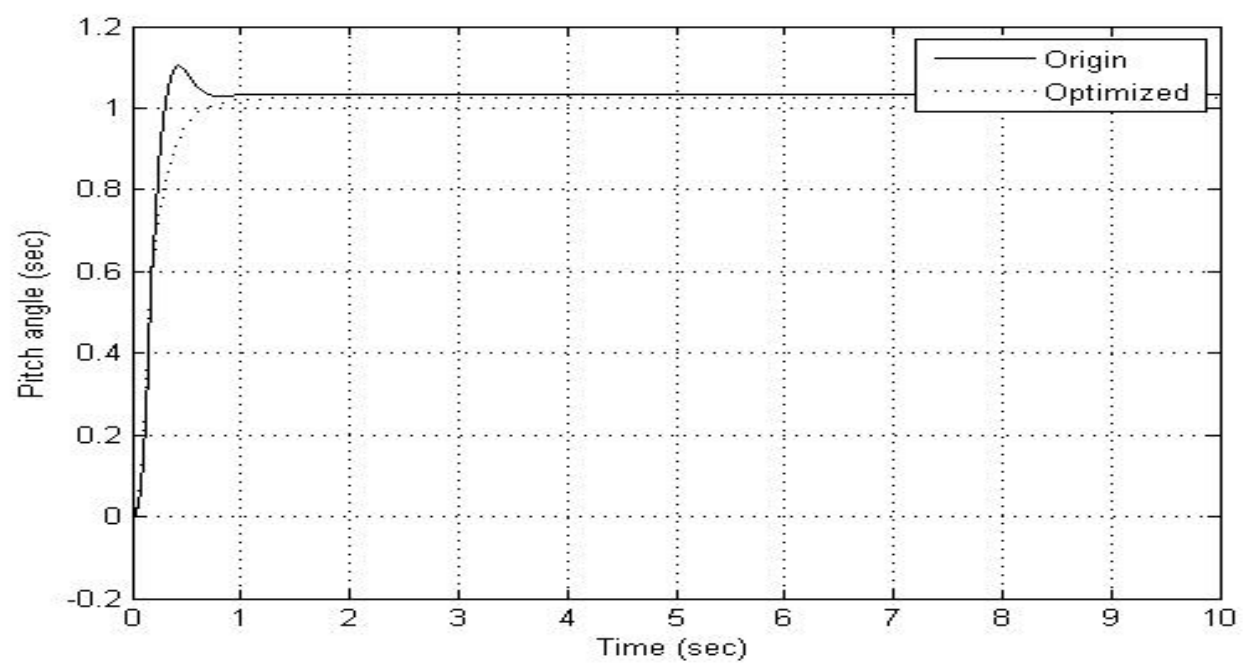

Figure (5): Missile response at $75 \mathrm{Sec}$ 


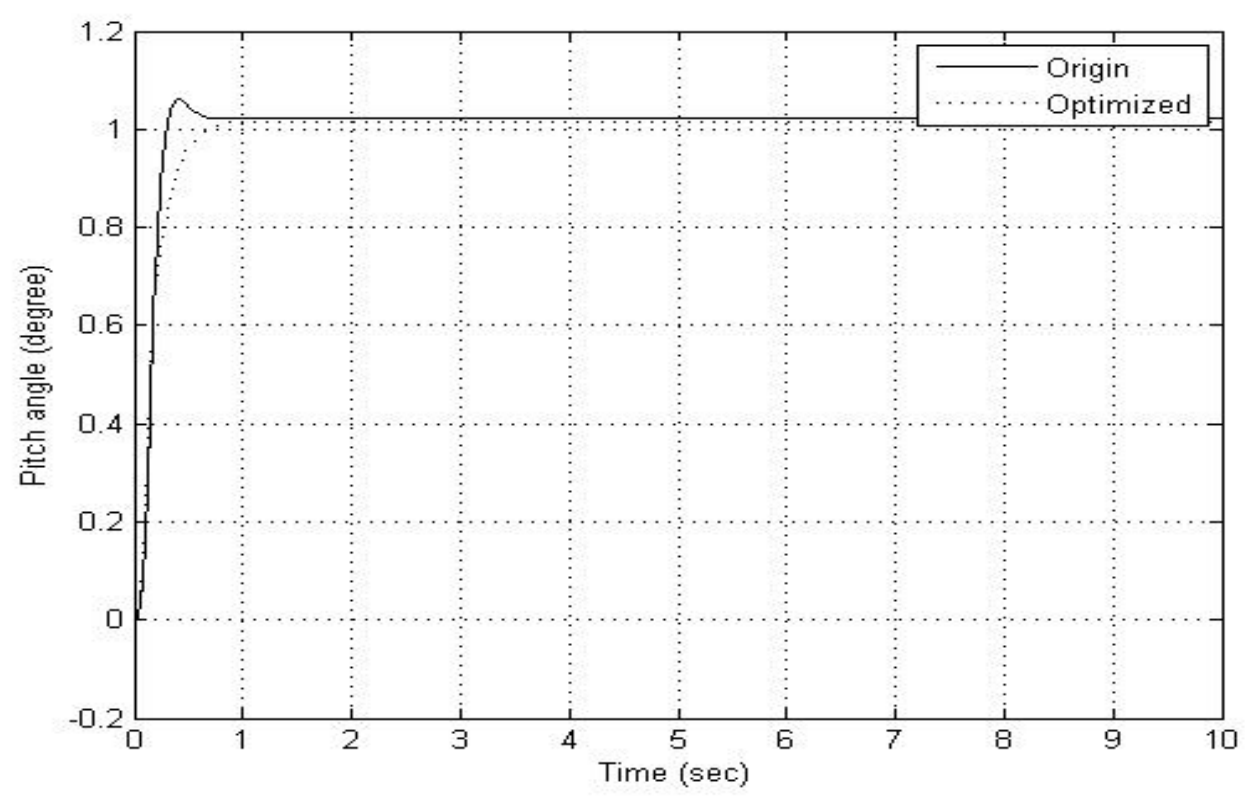

Figure (6): Missile response at 100.4 Sec

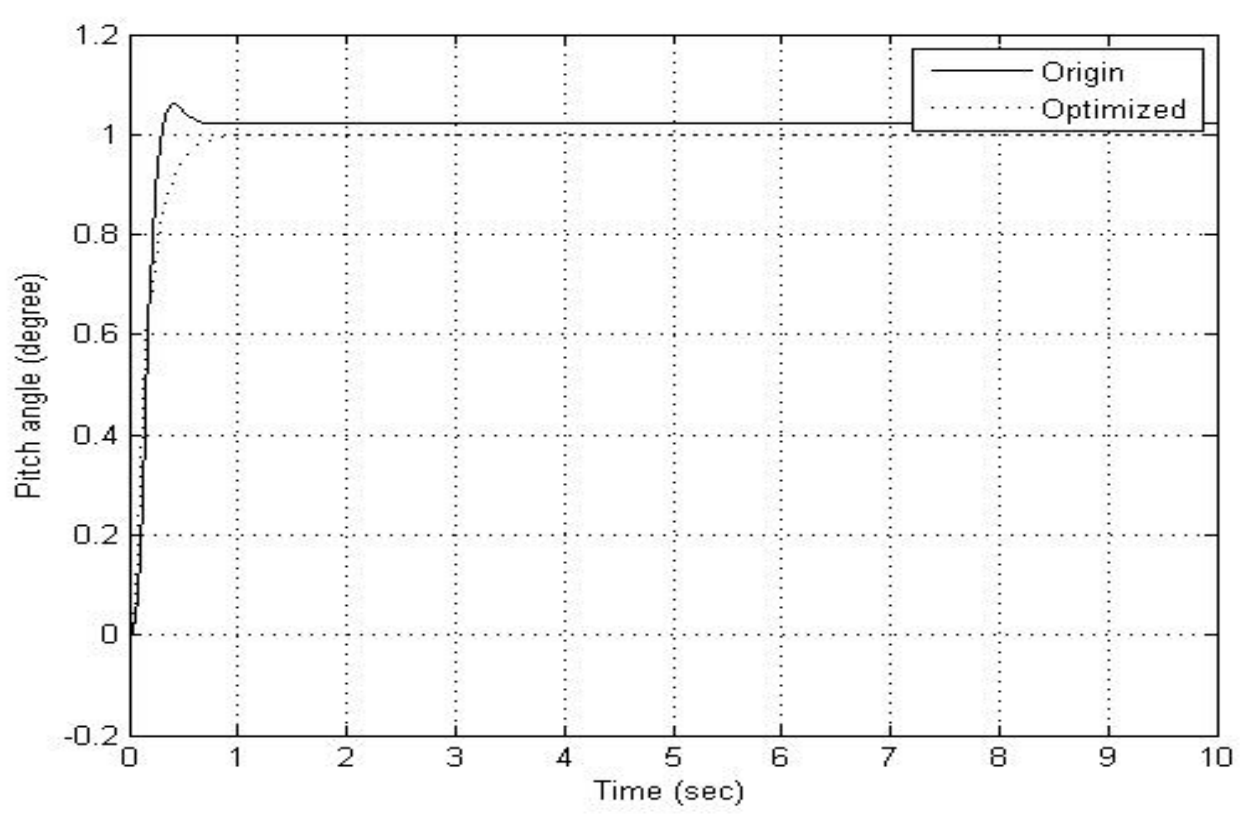

Figure (7): Missile response at $139 \mathrm{Sec}$ 


\section{Conclusion:}

In this paper, the work of a new self-tuning PID controller for ballistic missile has been presented. By using the method of gradient descent optimization to the parameter space, the PID parameters are adjusted automatically online. This makes the PID controller robust to changes in dynamic model. In addition, this control approach brings the pitch angle to a desired reference trajectory. Theoretical analysis and simulation results were presented to show the effectiveness of this simple approach.

\section{References:}

[1] K.J Astorm, T. Hogglund, "Automatic tuning of simple regulators with specification on phase and amplitude margins," Automatica, 20, 1984, pp.645651.

[2] K.J Astorm, T. Hogglund, "Towards intelligent PID controller," Automatica, 28, 1992, pp.1-9.

[3] F. Lin, R. D. Brandt, G. Saikalis, "Self Tuning of PID controller by adaptive interaction," Proceeding of the American control conference, June 2000, pp.3676-3681.

[4] J. A. Snyman "Practical mathematical optimization," Springer, 2005.

[5] Y. Tan, C. Yi Su, "Feedback control technique for gradient based learning," IEEE Transactions on Automatic Control, No. 2, April 2000.

[6] J. H. Blakelock, "Automatic control of aircraft and missile," John Wiley,1991

[7] M. R. Peresada S. Valigi "Adaptive input-output linearizing control of induction motor," IEEE Transaction on automatic control, 38, 1993,pp.208-221 